\title{
Experimental Investigation of the Hybrid Damper Clutch System Under Dynamic Conditions of an Automobile Powertrain
}

\author{
Mehmet Onur Genç ${ }^{* 1}$ (i), Süleyman Konakçı' ${ }^{1}$, Necmettin Kaya² ${ }^{(0)}$, Çağlar İmer ${ }^{1}$ (D), \\ Ali Kamil Serbest ${ }^{3}$
}

Valeo Automotive Systems, Bursa, Turkey.

²Bursa Uludağ University, Mechanical Engineering, Bursa, Turkey - necmi@uludag.edu.tr

${ }^{3}$ Angst-Pfister Advanced Technical Solutions, Bursa, Turkey - alikamil.serbest@angst-pfister.com

\begin{abstract}
Automobile components are subjected to high dynamic forces and vibrations under operational conditions, which need detailed system analysis for work properly. Clutch is one of the important parts of the automobile powertrain system with torque transmission controlling and vibration damping properties. Metallic helical springs are widely preferred within the clutch discs with their durable mechanic properties against dynamic variables on an automobile. Recently, the rubber components are used in the automotive industry due to some advantages such as wear reduction, cost etc. Therefore, in this study, the clutch system consists of metallic and rubber spring dampers are investigated to observe mechanical behaviours together in use. In order to investigate this hybrid damper behaviour under dynamic condition in clutch system, the torsional endurance was observed with the functional bench tests to simulate driving condition. Results show that the Hybrid dampers have a close durability performance within acceptable damper torque decrease found according to experimental vehicle test results. These results also shows that for medium vehicle segments, Hybrid damper is an alternative within the aimed vehicle comfort level. Hybrid clutch dampers are new in literature, and firstly were investigated in this study in terms of endurance capability.
\end{abstract}

Keywords: Hybrid damper, Rubber damper, Metallic damper, Hyper-viscoelastic modeling, Torsional fatigue, Clutch disc, Powertrain system, Vehicle comfort

\section{INTRODUCTIONS AND MAIN SECTIONS}

Automobile clutch system components are subjected to high dynamic forces under operational conditions. The clutch disc is in charge of damping the vibrations coming from the engine properly to improve driving comfort and transmitting the torque. Torque transmission role of a clutch disc is achieved with a damper system which consists of metallic springs conventionally.

A clutch disc transmits clamped force through the friction from pressure plate and flywheel (Figure 1). During gear shifting, drivers push the clutch pedal and clutch disc locates to disengagement position, which provides torque cutting to enable gear shifting. Torque (T) which is transmitted by friction between flywheel and pressure plate is proportional with clutch disc facing friction coefficient fs, clamp load $\mathrm{F}(\mathrm{N})$, a number of friction surface $\mathrm{N}$ and medium diameter of friction surface Rm (Eq.1).

$$
T=f_{s} F N R
$$

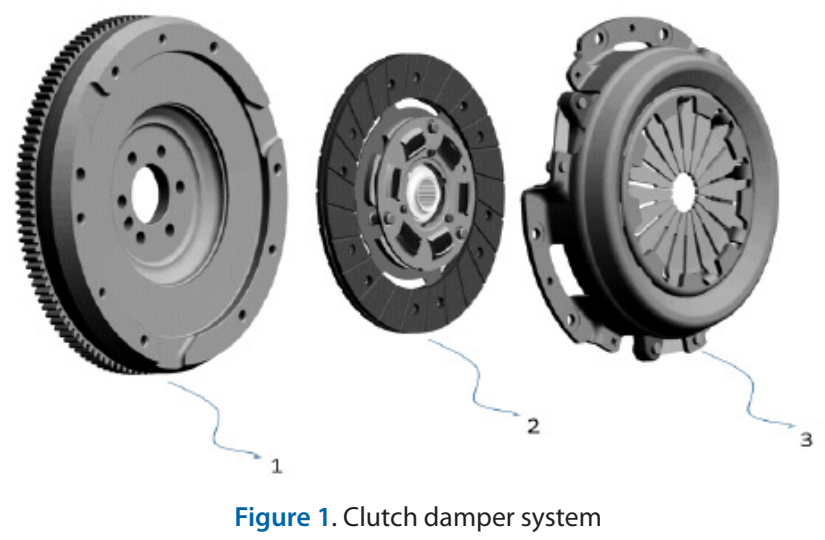

Under driving conditions between engine and transmission relative motions occur between engine and transmission due to dynamic variables. Relative motion between flywheel and clutch disc causes slippage that leads to dissipated energy. During slippage, thermal load in clutch house increases and this may cause permanent or temporary changes on materi-
* Corresponding author
Email: mehmetonurgenc@gmail.com
European Mechanical Science (2021), 5(2): 44-50

doi: https://doi.org/10.26701/ems.751149

Received: June 11, 2020

Accepted: December 29, 2020 
al properties of the components. The dissipated energy and wear on friction materials increase with slipping duration. Under normal working conditions, the amount of kinetic energy converted into heat leads to temperature increase. Generated heat flux during the slippage phase is distributed between flywheel and clutch based on their thermal diffusivity. The heat is transferred by conduction between the solid parts depending on their specific heat $\mathrm{Cp}[\mathrm{J} / \mathrm{kg} \mathrm{K}]$ and mass $\mathrm{m}[\mathrm{kg}]$ (Eq. 2).

$$
Q=M C p \Delta T
$$

Rubber materials have viscoelastic properties that need high attention before modeling such as 'Mullins effect', which is called as stress softening behavior of cross-linked rubbers at first cycles. Rubbers have variable hysteresis behaviors which change based on variable dynamic conditions apart from metallic springs. During rubber damper spring design, some countermeasures should be taken into account due to its viscoelastic properties, which subject to loss of stiffness under dynamic condition. Rubber based materials are accepted as incompressible and have a specific range of Poisson ratio and young modulus. Figure 2 shows the experimental compression test results of the standard test specimen measured in this study compared to metal spring. This graph explains the importance of stabilization process of rubbers for the chosen strain rate in the dynamical system. Rubbers show nonlinear load characteristics, and they are modeled with different kinds of material behaviors. Instead, metal springs tend to show same linear force reaction under compression.

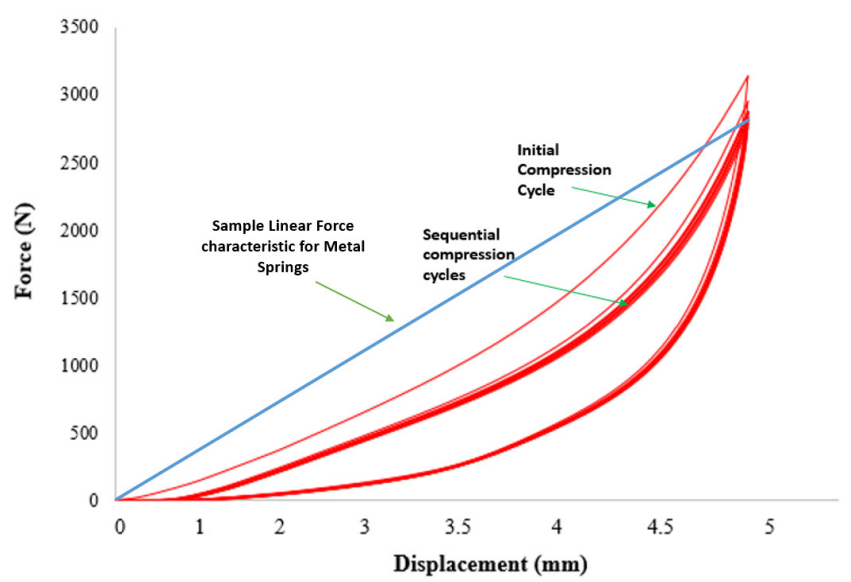

Figure 2. The experimental test of 'Mullins Effect' vs. Metal spring linear characteristic

There are both advantages and disadvantages of using rubber springs in automobile clutch systems compared to metal helical springs. The major advantage in using rubber springs is the low-cost. Besides that, since the internal hysteresis of the rubber springs is higher than the metal helical spring, under normal driving conditions, it is expected that the vibration amplitude transmitted from engine to gearbox may be lower with higher damping coefficient. As a disadvantage of rubber spring, damping torque loss occurs due to loss of stiffness within the time related to viscoelastic material behavior. In metal helical springs, the stiffness is more durable due to the metal material behavior during product lifetime. The hybrid damper system (Figure 3 ) is the new generation design for the clutch system technology, and in the near future, the usage of this damper type is expected to be increased. The system consists of metallic and rubber springs in the clutch disc.

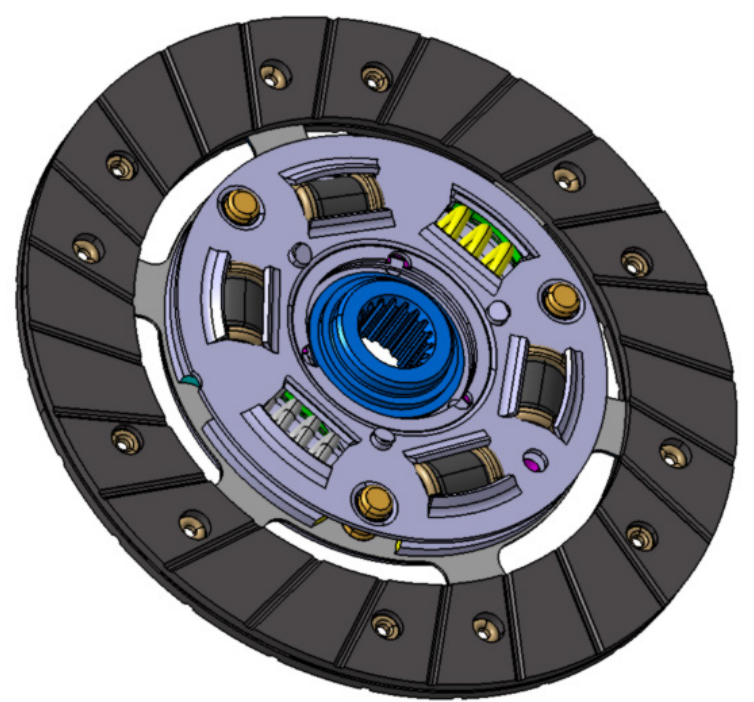

Figure 3. Hybrid damper clutch disc design

In the literature, rubber-based materials have been investigated with different approaches. Jadhav et al. [1] have studied the hyperelastic modeling of the mechanical behaviors of rubber materials, such as natural rubber and silicone, and rubber materials, ranging from muscles to biological materials such as skin texture, by hyperelastic modeling. Marvalova [2] experimentally measured the viscoelastic stress response by characterizing the mechanical behavior of rubber structures in deformation processes under isothermal conditions. Viscoelastic stress relaxation behaviors were investigated by examining the structural function parameters under variable frequencies. Zhang and Zhang [3] studied the viscoelastic parameters of rubber bushings and studied thermal analysis, which determines their physical properties by DMA (Dynamic Mechanical Analysis). A new approach to the prediction of hysteresis losses based on the interaction between molecular chains of polymer structures has been proposed. Bani et al. [4] observed the temperature of increased rubber-metal springs under loading conditions and observed heat accumulation due to hysteresis losses and low rubber thermal conductivity resulting from increased temperature. They categorize physical and chemical impairments as a result of heat accumulation, which is the main cause of aging in rubber, and point out that the increase in hardness and the decrease in damping ability. Zhang and Zhang [5] investigated the deterioration and the parameters of the thermal behavior of the rubber caused by the working conditions in the rubber bushes used in aviation or aviation to reduce noise and vibration. The data obtained at a different frequency and amplitude values are modeled by modeling the temperature-dependent change in viscoelastic behavior. Genç and Kaya [6] investigated the elastomer damper design under 
various conditions in their study. They used NBR rubber material in their rubber spring. According to their results, in case of full elastomer damper was used with NBR material, the clutch damper tents to loose $\% 15$ stiffness in first $10 \mathrm{~K}$ $\mathrm{km}$ driving. These results show the sensitive design necessity of rubber dampers in automotive clutch system due to stiffness losing issue.

The focus of this article is to investigate Hybrid Damper Clutch behaviors in automotive clutch system. In order to obtain the results, the same clutch design was produced with metal and rubber springs. The Hybrid damper systems have newness for its area, and during this study, the system was investigated and functional test results were obtained. Torsional endurance behavior is the most important parameter for the damper systems because the target damping level can be achieved in case the nearly constant damper stiffness level is obtained. Figure 4 explains the general work flow conducted in this study.

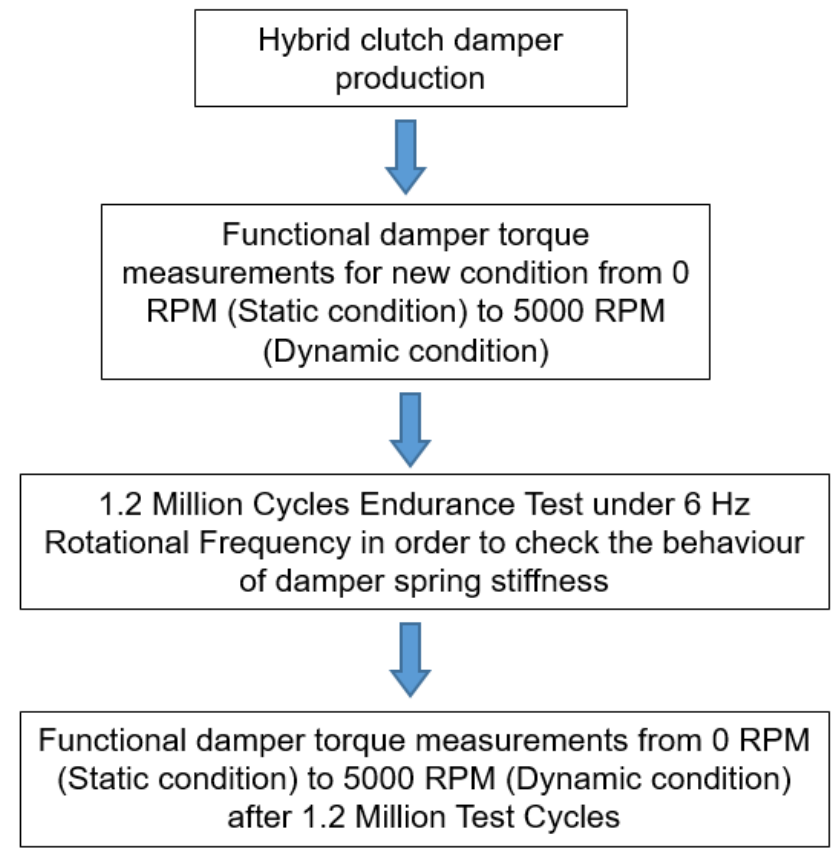

Figure 4. Workflow performed in the study

\section{MATERIAL AND METHODS}

In this section, the properties of the Hybrid Damper system and the test methods are investigated. Figure 5 shows the prototype of the Hybrid Damper consists of four (4) rubber-based springs and two (2) metallic springs. The geometry and the number of springs can be selected according to clutch house design and automobile properties. The damper system should be checked and verified in terms of system endurance and robustness.

In an automobile powertrain system, it is expected that there will be a loss of stiffness in the rubber damper spring during the life of the product in case of temperature, frequency compression, loss of length (creep). In parallel with these stiffness losses, the hysteresis (damping torque) varies due to internal friction changes. Due to these situations, it is expected that the filtering properties of the clutch damper will decrease in the following kilometers. These features can be obtained with sensors and data collection devices in long vehicle tests and NVH tests. With the loss of stiffness, vibrations are expected to be less dampened and the vibration amplitudes measured in the transmission are higher than in the past. These situations lead to a decrease in vehicle comfort and an increase in the level of vibration felt by passengers.

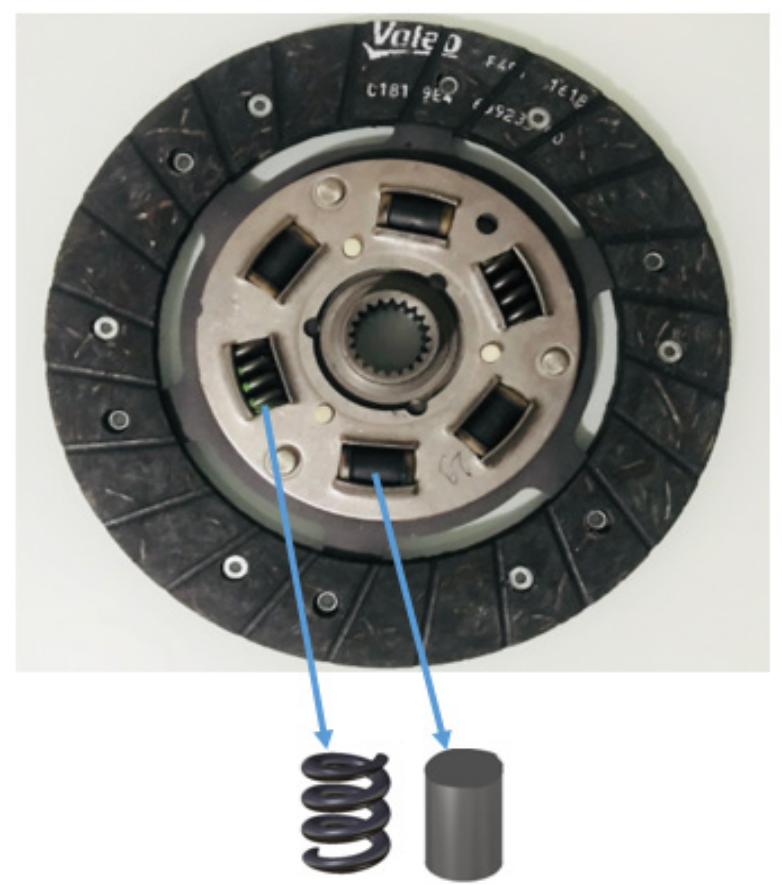

Figure 5. The prototype of hybrid damper clutch disc

Dynamic torsional endurance test simulates the worst condition on the automobile with representing the endurance of springs against torque oscillations generated by the engine. Many factors have high effects on the torque oscillations of the automobile such as driver profile, air conditioner on/off, lightening on/off, fuel type gasoline/diesel, fuel quality, engine maintenance quality, abusive usage, etc. In this study, the clutch discs with hybrid damper have been subjected to dynamic torsional fatigue compressions under various RPM (revolution per minute) with $6 \mathrm{~Hz}$ test frequency. Figure 6 shows the torsional compression on the clutch disc during the dynamic torsional endurance test. The test was performed at 1000, 2000, 3000, 4000 and 5000 RPM with a total $1.2 \mathrm{M}$ cycles. According to experimental vehicle test results obtained during driving condition, 1.2 Million cycles equal to $100 \mathrm{~K} \mathrm{Km}$ in real vehicle usage [7]. The target is to observe the torsional endurance including stiffness loose and wear behavior on the rubber springs. Conversely, the metallic springs are expected to keep its stiffness level during the test condition thanks to its material properties.

Figure 7 shows the compression and release phases of rubber springs on the torsional durability test bench. In this study, the clutch disc was compressed to $6,5^{\circ}$ angular travel and then released phase was started till to starting position. Hysteresis is the loss of damper torque that occurred due 
to internal friction in the hybrid damper. Clutch disc using rubber springs is fitted on "Dynamic torsional endurance test bench (Figure 8) with test flange providing relative motion at each cycle of test. The test machine consists of two main parts, one of them is the dynamic controlling screen, which enables to control mechanical test machine under option from $1 \mathrm{~Hz}$ to $6 \mathrm{~Hz}$. Second part is the mechanical test machine operates based-on the hydraulic forces. Disc assembly using rubber springs is fitted with test flange providing relative motion at each cycle of test. Test was performed up to $5000 \mathrm{RPM}$ (revolution per minute) at $6 \mathrm{~Hz}$ compression frequency. During the rotations from 0 RPM to 5000 RPM, the hybrid clutch damper is forced to withstand $6 \mathrm{~Hz}$ oscillations which simulates possible endurance performance during driving conditions on the road. As a result of this functional bench test, the endurance performance under dynamic conditions can be predicted.

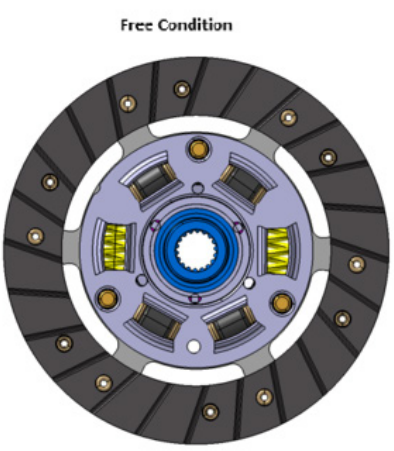
$\begin{array}{ll}\text { a) Free Condition } & \text { b) Compressed Condition }\end{array}$ Clutch disc compressed condition on the test bench

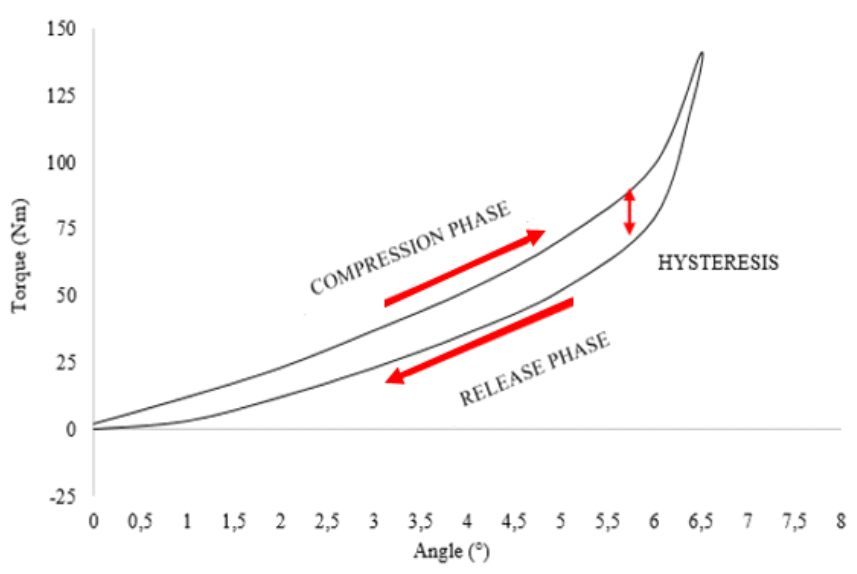

Figure 7. Compression and release phases of rubber springs

Figure 9 shows the dynamic condition effect on damper springs. Centrifuge effect is expected to increase in parallel to RPM. By means of centrifuge effect, measured damper torque is increased because of friction force rising between damper springs and the metallic components such as retainer plate, drive plate of the clutch damper disc.

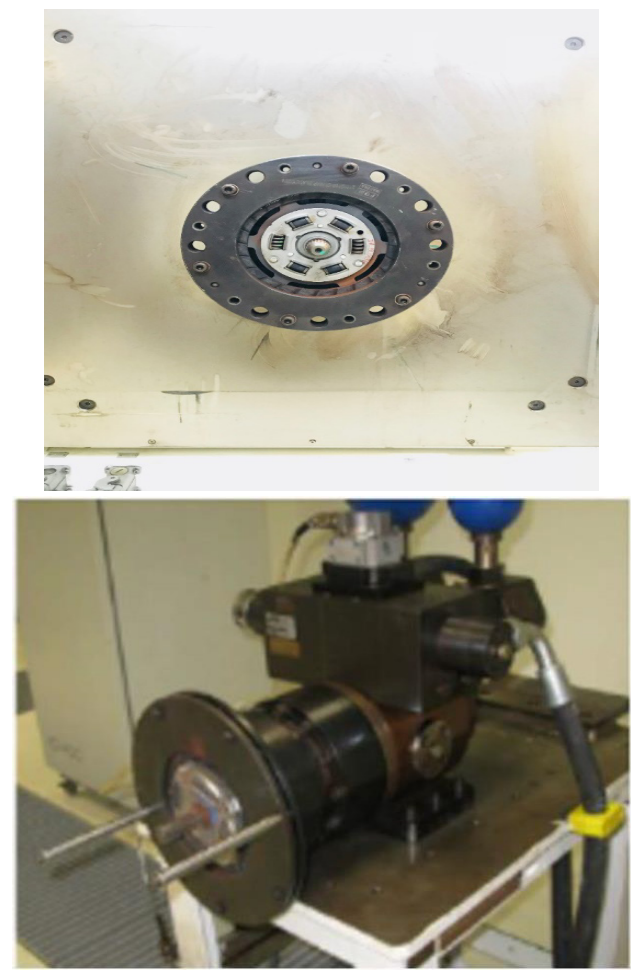

Figure 8. Dynamic torsional endurance test bench

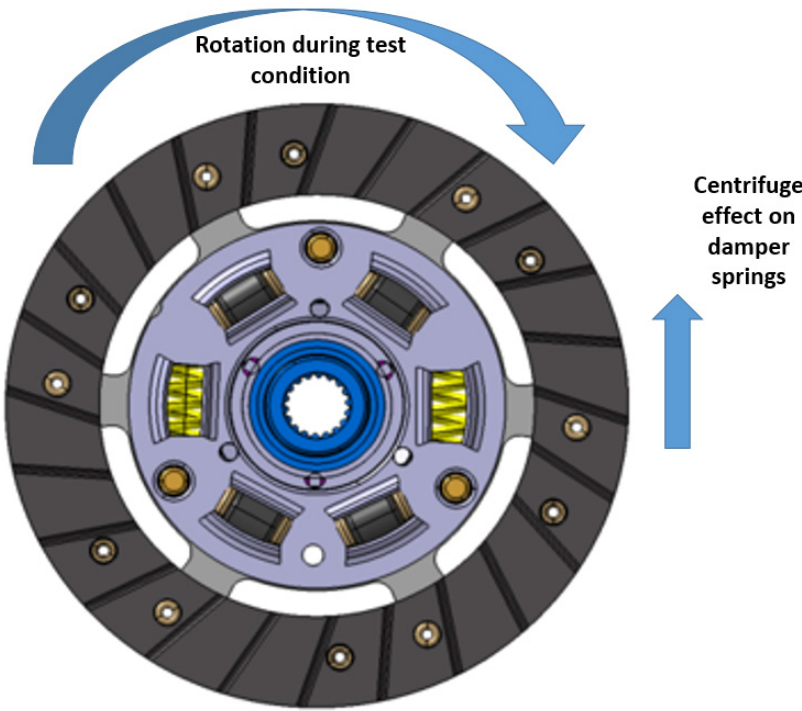

Figure 9. Centrifuge effect on damper torque behavior

\section{ANALYSIS AND DISCUSSIONS}

\subsection{Experimental Results}

In this section, the dynamic torsional endurance test performed to hybrid damper clutch disc is observed and the results are evaluated. Figure 10 shows the torsional damper curve prior to the dynamic functional curve. The graph explains the dynamic damper torque of the hybrid damper system. The damper torque graph shows that the dynamic torque of the clutch damper system tends to increase during the RPM value is drastically increased. Table 1 indicates the detailed values for each RPM compared to measured torque level. 


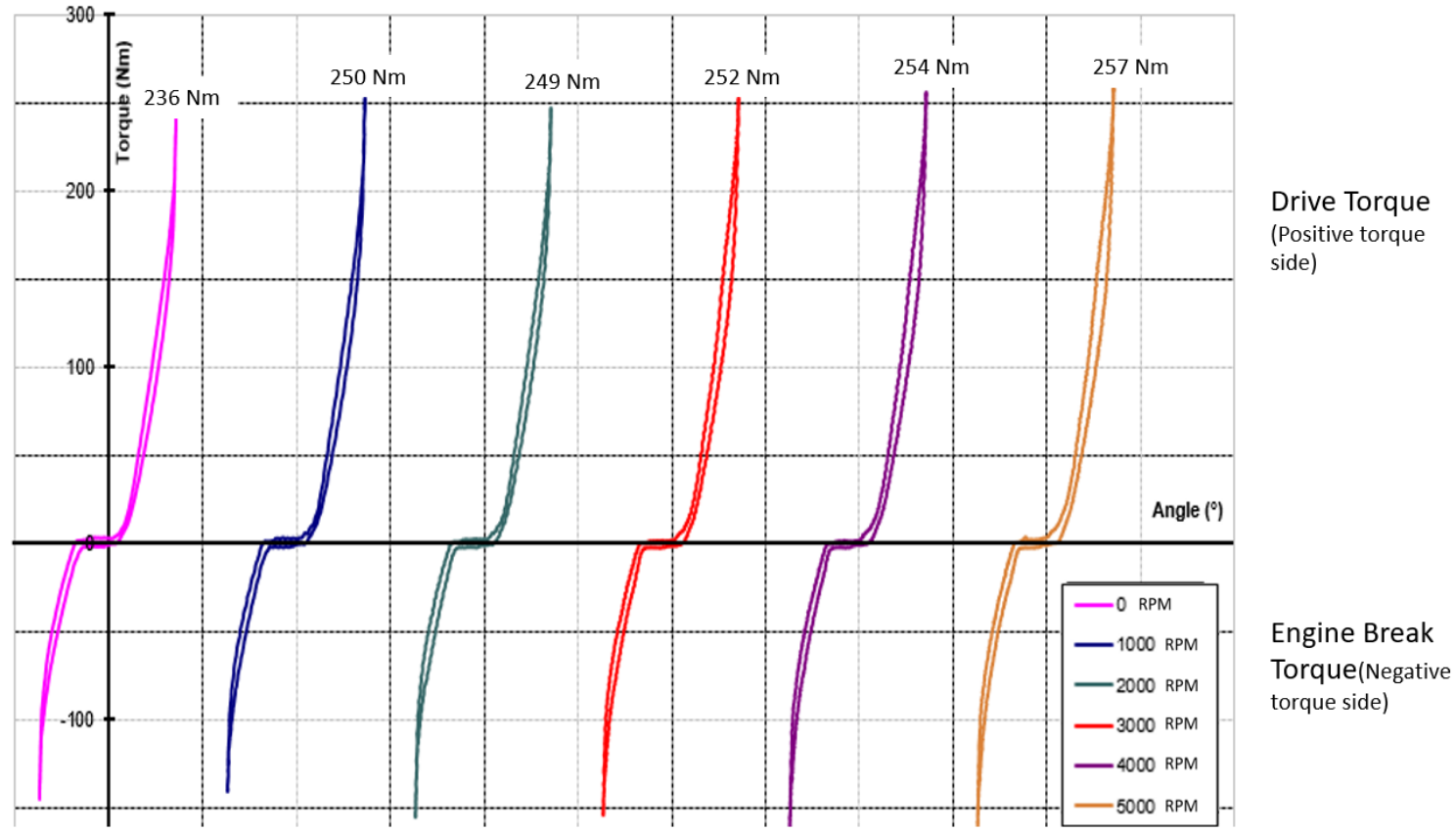

Figure 10. Dynamic torsional endurance test under $6 \mathrm{~Hz}$ \& damper torque measurements before the test under various RPM

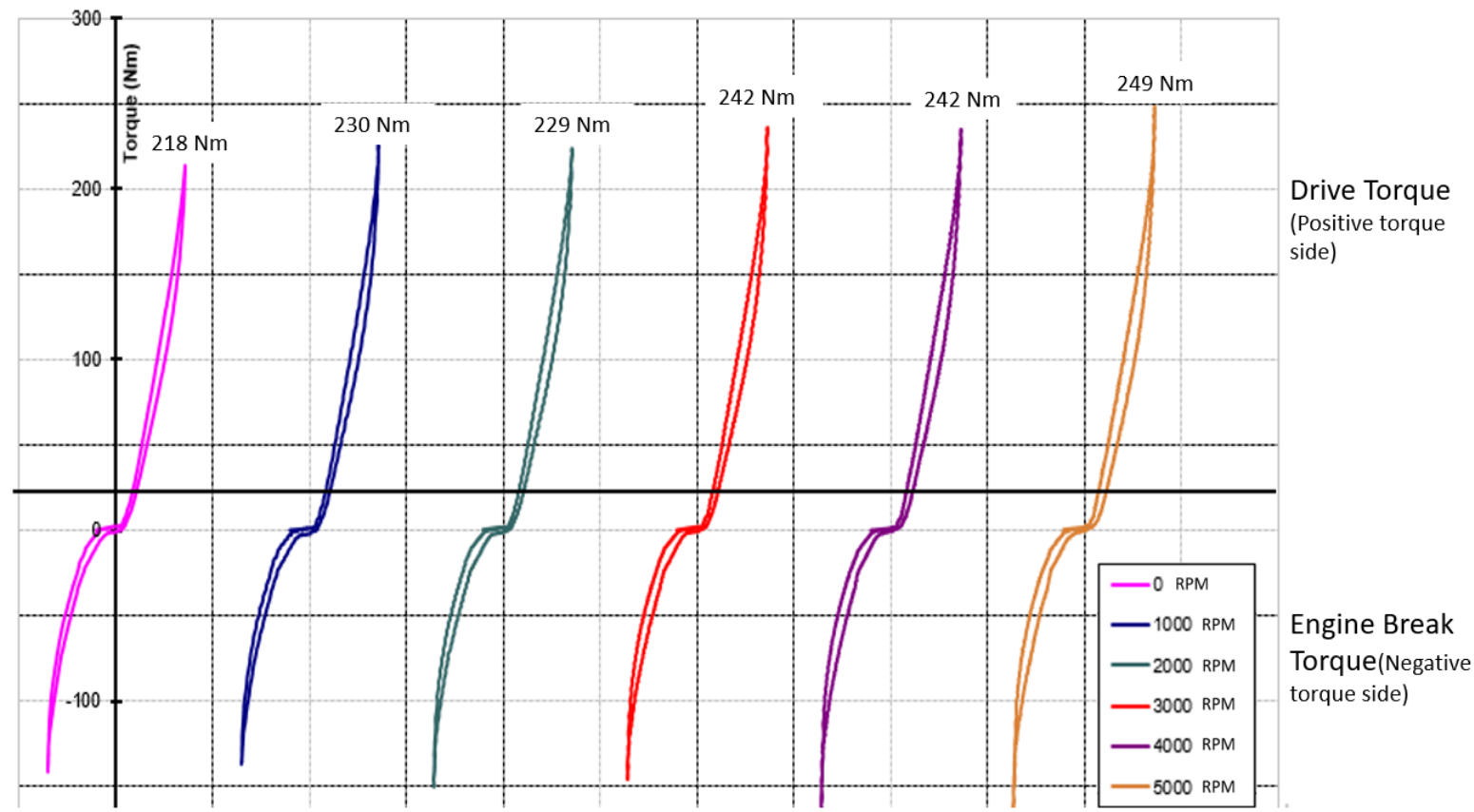

Figure 11. Dynamic torsional endurance test under $6 \mathrm{~Hz}$ \& damper torque measurements after $1.2 \mathrm{M}$ cycle under various RPM

Table 1. Measured dynamic torque under dynamic condition \& Initial Condition

\begin{tabular}{|c|c|}
\hline RPM (Revolution per minute) & Damper torque measured $(\mathrm{Nm})$ \\
\hline 0 & 236 \\
\hline 1000 & 250 \\
\hline 2000 & 249 \\
\hline 3000 & 252 \\
\hline 4000 & 254 \\
\hline 5000 & 257 \\
\hline
\end{tabular}

Figure 11 is the graph of dynamic torsional endurance test measured after 1.2 M cycle. Results show that the damper torque decrease is observed at the minimum level. This information shows the endurance level of the hybrid damper system is verified for the defined clutch disc design. Table
2 indicates the detailed values for each RPM compared to measured torque level after 1.2 M Cycle.

Table 2. Measured dynamic torque under dynamic condition \& After 1.2 M Cycle

\begin{tabular}{|c|c|}
\hline RPM (Revolution per minute) & Damper torque measured $(\mathrm{Nm})$ \\
\hline 0 & 218 \\
\hline 1000 & 230 \\
\hline 2000 & 229 \\
\hline 3000 & 242 \\
\hline 4000 & 242 \\
\hline 5000 & 249 \\
\hline
\end{tabular}

Table 3 is the comparison of the full metallic (conventional) damper system and hybrid damper system designed for the same vehicle application (Figure 12). Both design have same 
geometries and components except damper spring group. Hybrid damper system consists of four (4) rubber spring and two (2) metallic spring compared to full metallic system which has 6 metallic spring. The clutch type used in this study is of medium torque transmission capacity and used in B segment passenger vehicles. Figure 13 shows the comparable geometries of both springs. Due to envelope limits, metallic and rubber springs were designed for nearly same mechanical reaction forces, also this phenomena widely called as damper stiffness. The point that also needs attention is metallic spring production process has more steady geometrical tolerances, however the rubber springs have not this kind of stability due to injection process. This is also the disadvantages of rubber spring production process.

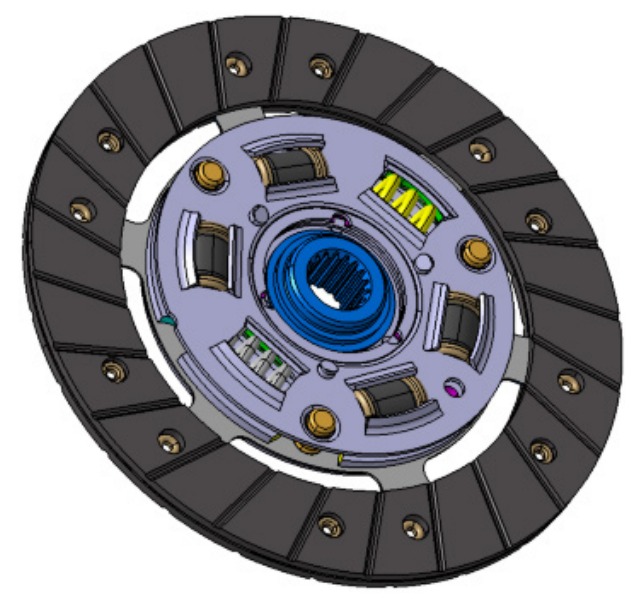

a) Hybrid damper system

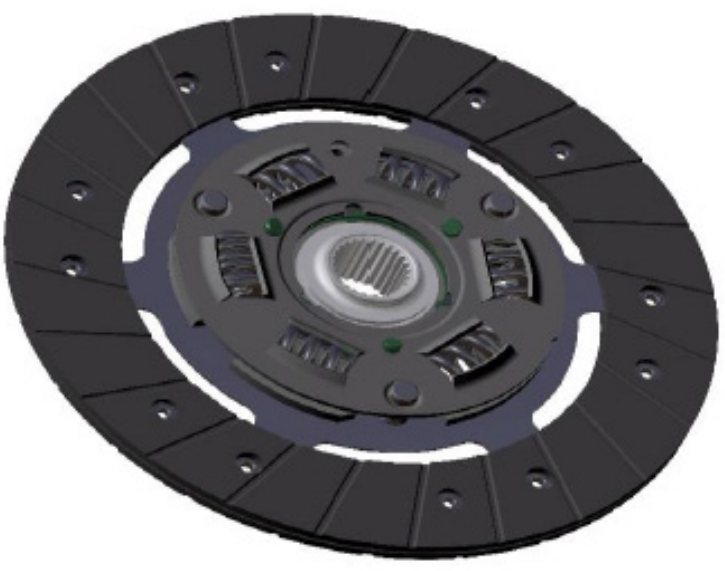

b) Full metallic damper system

Figure 12. Hybrid damper system design vs. Full metalic (conventional) damper system design

Metallic damper test was performed in Valeo Automotive plant [7]. Results show that the damper torque loss is observed about 3\% for the full metallic system, however, the hybrid damper system was observed about $8 \%$. This result explains that the hybrid damper system can be operated in a safe condition and the rubber damper inside the system can keep its stiffness level during the product life. These results show that full metallic spring usage creates more safety in terms of stiffness decrease compared to Hybrid damper system. However, damper torque decrease can tolerate the deviations up to $10 \%$ according to experimental test results on the road [7]. Nevertheless, in case full rubber spring uses instead of hybrid or full metallic system, more stiffness loose is expected which can create critical torque transmissiblity [6].

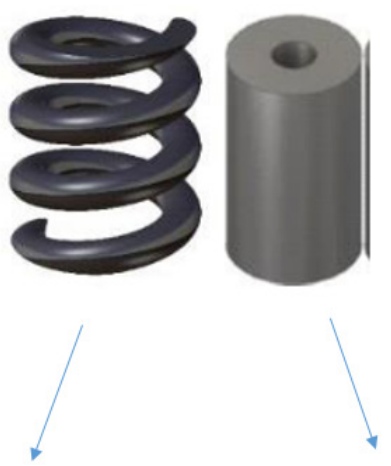

Metallic damper spring b) Rubber damper spring

Figure 13. Detailed view of metallic and rubber spring

Table 3. Damper torque comparison of Hybrid \& Full Metallic damper systems at static condition (0 RPM)

\begin{tabular}{|c|c|c|c|}
\hline & $\begin{array}{c}\text { Max damper } \\
\text { torque before } \\
\text { the test }(\mathrm{Nm})\end{array}$ & $\begin{array}{c}\text { Max damper torque after } \\
1.2 \text { M dynamic torsional } \\
\text { endurance test }(\mathrm{Nm})\end{array}$ & $\begin{array}{c}\text { Damper } \\
\text { torque loss } \\
\text { ratio (\%) }\end{array}$ \\
\hline $\begin{array}{c}\text { The full metallic } \\
\text { damper system [7] }\end{array}$ & 217 & 210 & 3 \\
\hline $\begin{array}{c}\text { Hybrid damper } \\
\text { system }\end{array}$ & 236 & 218 & 8 \\
\hline
\end{tabular}

\subsection{Vehicle comfort overview based on experimental results}

Vehicle comfort is one of the phenomena for vehicle clutch design. In literature, this condition is called as Noise, Vibration and Harshness (NVH). Generated torque transmission on powertrain system creates unwanted oscillations in the system, and clutch damper disc is one of the damping section showing in Figure 14. This 1-D model is prepared with LMS Amesim software with real vehicle configuration data. This schematic view works based on mathematical model, and this means that major deviations on damper stiffness more likely creates resonance changes on the sub-sections of the powertrain system such as Gearbox, Propeller Shaft etc $[13,14]$. For the vibration analysis of rotational components, as in vehicle powertrain system, angular frequency should be taken into account which has directly linked with stiffness ( $k$ ) in mathematical condition. Therefore, in case the performance reliability of vehicle comfort is compared in sequence for all three (3) conditions, full metalic damper has consistent better NVH performance than the Hybrid and Rubber dampers due to less stiffness decrease within the time. However, Hybrid dampers have average stiffness loose, and it can be obtained from the experimental results that Hybrid damper system can be preferred in most cases for medium segment vehicles such as B Segment and low torque C Segments (Table 3). 


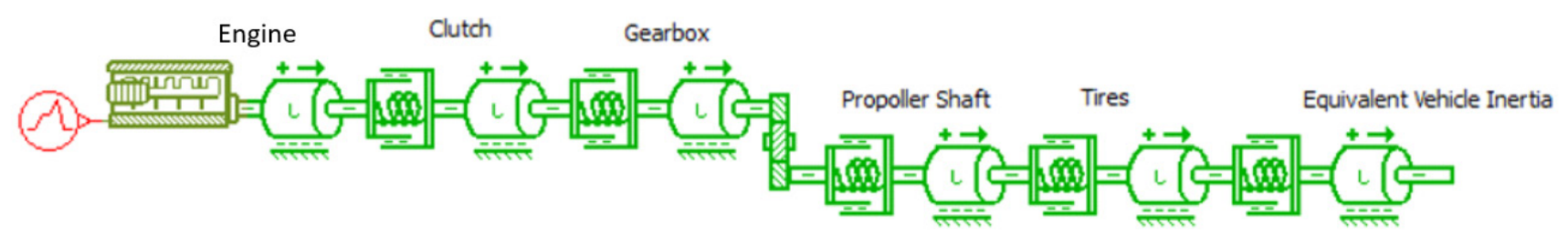

Figure 14. 1-D Modeling of vehicle powertrain system for designed Hybrid damper clutch

\section{CONCLUSIONS}

This study investigates the design of the hybrid damper system under dynamic conditions verified with dynamic torsional endurance test. In the study, the produced samples of hybrid damper clutch disc were subjected to dynamic torsional endurance test comparatively with a full metallic clutch damper system. The results show that the hybrid damper system design loses its damper torque level averagely $8 \%$ after $1.2 \mathrm{M}$ cycles which equals $100 \mathrm{~K} \mathrm{Km}$ vehicle usage in real driving condition, however, the conventional full metallic damper system design loses $3 \%$ with the same test condition. These results show that the hybrid damper system design is safe because the damper torque loss is under $10 \%$, which is accepted as safety limit. During driving conditions, the vehicle is supposed to show variant driving profiles which results in different product life with small deviations. The functional bench test results obtained in this study simulate the real driving condition, according to results, hybrid damper systems can be an alternative solution of full metallic damper clutches in automotive industry.

\section{Acknowledgements}

The authors gratefully acknowledge the support of TUBITAK under grant the 3180181 project ongoing with collaboration between Valeo Automotive, Angst-Pfister and Bursa Uludag University.

\section{REFERENCES}

[1] Jadhav, N., Bahulikar, S.R., Sapate, N.H. (2016). Comparative Study of Variation of Mooney-Rivlin Hyperelastic Material Models under Uniaxial Tensile Loading. Publishing Corporation Scientific World Journal, Vol.2, Issue.4, IJARIIE-ISSN(O)-2395-4396.

[2] Marvalova, B. (2007). Viscoelastic Properties of Filled Rubber. Experimental Observation and Material Modelling. Engineering Mechanics, Vol.14, Issue.1/2, pp.81-89.

[3] Zhang, Z., Zhang, H. (2014). Viscoelastic Parameter Identification based Structure-Thermal Analysis of Rubber Bushing. Global Journals of Research in Engineering, Vol.14, Issue.3, Online ISSN: 22494596, Version 1.0

[4] Bani, M. S., Stamenkovi, D.S., Miltenovi, V.D., Milosevic, M.S., Miltenovi, A.V., Djeki, P.S., Rackov, M.J. (2012). Prediction Of Heat Generation In Rubber Or Rubber-Metal Springs. Thermal Science, Vol. 16, Issue. 2, pp. 527-539.

[5] Zhang, Z., Zhang, H. (2016). FEA based Dissipation Energy and Temperature Distribution of Rubber Bushing. International Journal of Engineering Research and Applications, ISSN: 2248-9622, Vol.6, Issue.1, (Part - 2), pp.48-56.
[6] Genc, M. O., Kaya, N. (2020). Design and verification of elastomer spring damping system for automobile powertrain systems. Journal of the Faculty of Engineering and Architecture of Gazi University 35:4, pp. 1957-1971.

[7] Valeo Automotive Systems, (2019). Vehicle Test Technical Catalogue, Bursa, Turkey.

[8] Melnik, R.V.N, Strunin, D.V., Roberts, A.J. (2005). Nonlinear Analysis of Rubber-Based Polymeric Materials with Thermal Relaxation Models. Numerical Heat Transfer, Part A, pp. 549-569.

[9] Pacheco, J. L., Bavastri, C.A. , Pereira, J.T. (2015). Viscoelastic Relaxation Modulus Characterization Using Prony Series. Latin American Journal of Solids and Structures, Vol:12, pp. 420-445.

[10] Monsia, M. D. (2011). A Simplified Nonlinear Generalized Maxwell Model for Predicting the Time-Dependent Behavior of Viscoelastic Materials. World Journal of Mechanics, Vol. 1, Issue.3, pp. 158-167.

[11] Mohammed, M.A. (2014). Visco-Hyperelastic Model for Soft Rubber-like Materials. Sains Malaysiana, Vol: 43(3), pp. 451-457.

[12] Hwang, S. J., Chen, J. S., Liu, L., Ling, C. C. (2000). Modelling and Simulation of a powertrain system with automatic transmission. International Journal of Automobile Design, Vol. 23, No:1, DOl:10.1504/ IJVD.2000.001888

[13] Macor, A., Benato, A., Rossetti, A., Bettio, Z. (2017). Study and Simulation of a Hydraulic Hybrid Powertrain. 72nd Conference of the Italian Thermal Machines Engineering Association, ATI2017, Lecce, Italy.

[14] Genc, M. O., Kaya, N. (2018). Modelling and Experimental Investigation of Clutch Damper Spring Stiffness on Truck Driving Comfort. International Journal of Advances on Automotive and Technology, Vol.2, No.2, pp.121-136.

[15] Wu, H., Wu, G. (2016). Driveline Torsional Analysis and Clutch Damper Optimization for Reducing Gear Rattle. Shock and Vibration, Article ID 8434625, http://dx.doi.org/10.1155/2016/8434625.

[16] Chen, X., Wu, G., Wu., H. (2016). The Nonlinear Characteristics Impact of Multi-Staged Stiffness Clutch Damper on the Automobile Creeping. SAE 2016 World Congress and Exhibition, DOI: 10.4271/2016-01-0431. 